\title{
PERSPECTIVAS DA NOTIFICAÇÃO OBRIGATÓRIA DE DOENÇAS AO SERVIÇO VETERINÁRIO OFICIAL
}

\section{PERSPECTIVES FOR COMPULSORY NOTIFICATION OF DISEASE TO THE OFFICIAL VETERINARY SERVICE}

Ana Caroline Barros Correia do Monte ${ }^{\text {|* }}$

Resumo. Aproximadamente $75 \%$ das doenças infecciosas que afetam os humanos são de origem zoonótica. A saúde e a segurança das gerações animal e humana dependem, em parte, da nossa capacidade contínua de detectar, monitorar e controlar doenças e zoonoses emergentes ou reemergentes. O objetivo nesta revisão foi sintetizar as perspectivas da notificação obrigatória de doenças ao Serviço Veterinário Oficial (SVO), incluindo os instrumentos juridicamente vinculativos, a evolução e o status atual da notificação de doenças animais ao nível nacional, os benefícios e mecanismos da notificação oportuna e os fundamentos para medidas de segurança e controle de doenças. A Instrução Normativa $n^{\circ} 50$, de 24 de setembro de 2013, dispõe sobre uma lista de doenças de notificação obrigatória (DNO) no Brasil. A lista contempla, atualmente, um total de 141 doenças, incluindo as DNO para a World Organisation for Animal Health (OIE). Das doenças da lista da OIE no Brasil, 43 estão presentes ou com infecção limitada a uma, ou mais zonas. Na base de dados zoossanitários do Brasil, apenas 13 doenças tiveram casos notificados nos últimos 3 anos disponíveis (2017, 2018 e 2019). Pesquisas indicam elevado índice de subnotificação e falta de conhecimento sobre o processo de notificação. Instruções para notificação foram aqui apresentadas. A conexão entre a saúde humana, animal e ambiental, e a necessidade de estudar as doenças em seu contexto biológico, ecológico, médico e econômico, são apontados como mecanismo para prevenção e controle de doenças, de forma a promover e garantir a saúde nacional e globalmente.

PALAVRAS-CHAVE: Zoonoses. Doenças Emergentes. Saúde Ambiental.

Abstract. Approximately $75 \%$ of infectious diseases that affect humans are zoonotic. The health and safety of animals and human generations partialy rely on our continuous ability to detect, monitor and control diseases and emerging or re-emerging zoonoses. The purpose of this review was to summarize the prospects for mandatory disease notification to the Official Veterinary Service (SVO), including legally binding instruments, the evolution and current status of animal disease notification at the national level, the benefits and mechanisms of timely notification and the foundations for safety measures and disease control. The Normative Instruction $\mathrm{n}^{\circ} 50$, of September 24, 2013, provides a list of diseases of compulsory notification (DCN) in Brazil. The list currently includes a total of 141 diseases, including DCNs for the World Organization for Animal Health (OIE). From the OIE listed diseases, in Brazil, 43 are present or with infection limited to one or more zones. In the Brazilian animal health database, only 13 diseases reported cases in the last 3 available years (2017, 2018 and 2019). Research indicates a high rate of underreporting and a lack of knowledge about the notification process. Notification instructions have been presented here. The connection among human, animal and environmental health and the need to study diseases in their biological, ecological, medical and economic context are pointed out as a mechanism for disease prevention and control, in order to promote and guarantee national and global health.

KEYWORDS: Zoonoses. Emerging Diseases. Environmental Health. 


\section{INTRODUÇÃO}

Cerca de $75 \%$ das doenças que surgiram e afetaram humanos nos últimos dez anos foram causadas por patógenos zoonóticos, ou seja, patógenos que podem ser transmitidos de animais (principal reservatório da doença) para humanos. ${ }^{1}$ A maioria das doenças zoonóticas emergentes, incluindo influenza aviária altamente patogênica, vírus Nipah, severe acute respiratory syndrome (SARS), COVID-19 e pH1N1 (H1N1 pandêmico) causaram infecções graves e mortes em humanos em todo o mundo. ${ }^{2}$ Em relação aos rebanhos agropecuários, diversas doenças animais, tais como tuberculose bovina, anemia infecciosa equina, brucelose e raiva, reduzem a produção e produtividade animal e limitam o consumo e comercialização, causando prejuízos financeiros e riscos à saúde humana.3,4,5

No contexto da globalização e do crescente comércio internacional de animais e produtos animais, a notificação precisa, completa e oportuna da presença de doenças animais desempenha um papel crucial no comércio internacional e na saúde global. ${ }^{6}$ A detecção inicial de qualquer surto de doença em animais permite que os países em risco tomem as medidas adequadas para prevenir a propagação transfronteiriça da doença ou minimizar o seu impacto e, assim, mitigar os riscos associados à saúde animal e pública. ${ }^{1,5}, 6$

A World Organisation for Animal Health (OIE) é responsável por compartilhar, de forma rápida e transparente, informações precisas e completas sobre a situação global da saúde animal.7 Para tanto, a OIE coleta, verifica, valida e divulga as informações de saúde animal, incluindo informações sobre zoonoses, fornecidas por seus Integrantes designados pelos Governos de todos os Países Membros.5,6 No Brasil, o Sistema
Nacional de Informação Zoossanitária (SIZ), que engloba o Sistema Brasileiro de Vigilância e Emergências Veterinárias (SisBraVet), é o principal órgão do Serviço Veterinário Oficial (SVO) responsável por coletar, elaborar e divulgar informações zoossanitárias para subsidiar a elaboração, implantação, avaliação e tomada de decisões sobre estratégias e ações de vigilância, prevenção, controle e erradicação de doenças animais de relevância para a pecuária e para a saúde pública. ${ }^{8}$

A Instrução Normativa MAPA $n^{\circ} 50$, de 23 de setembro de 2013, estabelece uma lista de doenças de notificação obrigatória ao SVO. 9 A notificação da suspeita ou de casos confirmados de qualquer doença dessa lista é obrigatória para todos aqueles que tem conhecimento do caso, conforme os critérios e fluxos estabelecidos na norma. Portanto, os dados e informações que alimentam o SIZ são oriundas do próprio SVO, mas também de outros setores ligados à saúde pública, meio ambiente, ensino, pesquisa, laboratórios, iniciativa privada, médicos veterinários autônomos, técnicos, proprietários e produtores. $^{8}$

A subnotificação é regularmente citada como uma das principais limitações dos sistemas nacional e internacional de controle e prevenção de doenças. 5,6 Essa subnotificação pode estar associada as limitações de diagnóstico, a falta de informações sobre o processo de notificação, receio de perdas econômicas e falta de interesse em cooperar dentro da rede sociotécnica. ${ }^{1}$ Mais do que nunca, os serviços veterinários em todo o mundo enfrentam a necessidade de cumprir um papel crucial na proteção do estado de saúde animal de seus países, fornecer informações de vigilância confiáveis sobre a ocorrência de doenças em seus territórios e realizar análises de risco 
cientificamente válidas para se estabelecer no comércio internacional. ${ }^{10,3}$

O objetivo nesta revisão foi sintetizar as perspectivas da notificação obrigatória de doenças ao SVO, incluindo os instrumentos juridicamente vinculativos, a evolução e

\section{RESULTADOS E DISCUSSÃO}

Animais domésticos e silvestres atuam como reservatórios de doenças zoonóticas. $^{2}$ Animais domésticos, como gado, animais de estimação e aves, transmitem patógenos frequentemente aos humanos devido à interação próxima. ${ }^{11,12}$ Os roedores são muito abundantes, de natureza peri-doméstica e contribuíram com mais de 80 patógenos zoonóticos para os humanos. ${ }^{12}$ Os morcegos, por sua vez, foram identificados como hospedeiros reservatórios para muitas doenças emergentes em humanos, como Nipah, MERS-CoV, SARS, Rabies e Ebola. ${ }^{13}$ Os principais patógenos de doenças animais, incluindo zoonoses, são microparasitas, macroparasitas, vírus, bactérias, protozoários, vermes, carrapatos ou pulgas. ${ }^{14}$ Aproximadamente $20 \%$ de todas as zoonoses são transmitidas para o homem através de vetores, tais como mosquitos, carrapatos, moscas, flebotomíneos, pulgas, triatomíneos e caracóis aquáticos de água doce. ${ }^{15}$

As doenças em animais estão mudando em termos de espaço e hospedeiro. Subjacentes a isso, estão as mudanças demográficas e climáticas e uma demanda crescente por produtos de origem animal. ${ }^{16}$ Nos países ricos, as doenças endêmicas apresentem declínio histórico. No entanto, os países em desenvolvimento apresentam estabilidade ou deterioração da saúde animal e, em alguns casos, as doenças epidêmicas mostram expansão. ${ }^{17,5} \mathrm{~A}$ capacidade de o status atual da notificação de doenças animais em nível nacional, os benefícios e mecanismos da notificação oportuna e os fundamentos para medidas de segurança e controle de doenças.

detectar e relatar doenças de notificação obrigatória é limitada no Brasil e em muitos outros países. ${ }^{18,6}$ Essas limitações dificultam a avaliação das tendências, ocasionam prejuízos e colocam em risco a segurança alimentar e a saúde pública.

Doenças de notificação obrigatória: cenário histórico e atual

Muitas doenças de notificação obrigatória apresentam alta mortalidade e, em regiões mais pobres, onde o uso de tecnologias de produção agropecuária é limitado, podem destruir rapidamente rebanhos de animais agrícolas. ${ }^{5}$ Essas doenças, geralmente, reduzem a produção e produtividade, limitam o consumo e a comercialização local e exportações, causam prejuízos financeiros e riscos à saúde humana.3,4,5,6

A lista de doenças animais de notificação obrigatória em nível nacional está disponível no site do Ministério da Agricultura, Pecuária e Abastecimento.7 Há um total de 141 doenças de notificação obrigatória, contemplando os casos em abelhas, aves, bovinos e bubalinos, camelideos, equídeos, lagomorfos, ovinos e caprinos, suínos e múltiplas espécies. Essas doenças são classificadas em quatro grandes categorias, conforme o prazo de notificação:

Categoria 1 - 37 doenças erradicadas ou nunca registradas no Brasil, que requerem 
notificação imediata de caso suspeito ou diagnóstico laboratorial;

Categoria 2 - 17 doenças que requerem notificação imediata de qualquer caso suspeito;

Categoria 3-11 doenças que requerem notificação imediata de qualquer caso confirmado; e

Categoria 4 - 76 doenças que requerem notificação mensal de qualquer caso confirmado.

De acordo com os dados da Coordenação de Informação e Epidemiologia ${ }^{19}$, houve uma redução de $36 \%$ no número casos notificados nos últimos 10 anos, considerando todas as doenças (Figura 1). Em 2004, foi observado o maior número de casos de doenças da história da notificação no Brasil ( 94.050 casos). Destes, $86 \%$ foram casos de brucelose (Brucella abortus). A brucelose é uma doença zoonótica, causada por bactérias do gênero Brucella, considerada uma das doenças mais importantes dos bovinos. É uma doença endêmica no Brasil e com relatos em outros 127 países, afetando várias espécies de ruminantes, incluindo animais selvagens. ${ }^{20}$

O grande número de casos dessa doença no Brasil pode estar relacionado com tamanho do rebanho nacional, que é o maior do mundo. ${ }^{21}$

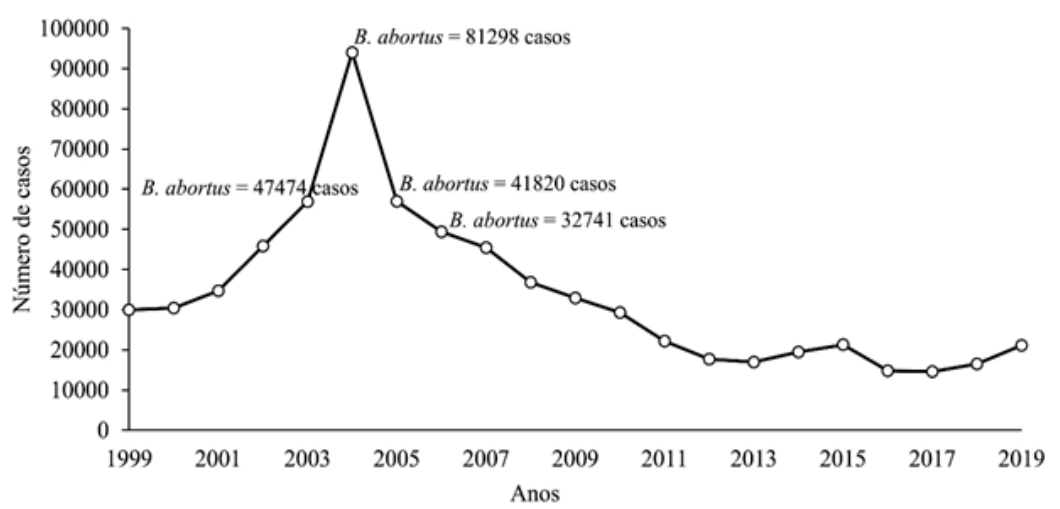

FIGURA 1: Distribuição temporal do número de casos das doenças animais no Brasil desde 1999. Fonte: MAPA 19

Considerando os dados dos últimos três anos disponíveis no SIZ, o número de casos notificados indica estabilidade temporal das doenças animais no país, incluindo brucelose e outras doenças importantes. Apenas 13 doenças têm sido notificadas em todo o território nacional (Tabela 1). Dentre estas, as cinco doenças com maior notificação média no país são a tuberculose bovina ( 5553 casos), anemia infecciosa equina (5268 casos), brucelose (4745 casos), raiva (1175 casos) e peste suína clássica (779 casos).

A subnotificação pode ser a principal responsável pela redução no número de casos notificados para algumas doenças. ${ }^{1,22}$ Pesquisas indicam que subnotificação pode estar associada as limitações de diagnóstico, a falta de informações sobre o processo de notificação, receio de perdas econômicas e falta de interesse em cooperar dentro da rede sociotécnica. ${ }^{1}$ No Brasil, pesquisas sobre os fatores subjacentes à subnotificação de doenças animais são escassas. Estimase que $69 \%$ dos profissionais da Medicina Veterinária, incluindo professores, estudantes e veterinários autônomos, não sabem como fazer a notificação obrigatória de doenças ao SVO. ${ }^{18}$

Algumas doenças endêmicas no Brasil, tais como B. abortus e a Leptospira spp., nem sempre provocam o desenvolvimento de sinais clínicos de infecção nos animais. ${ }^{23}$ 
A presença de animais assintomáticos e/ ou soronegativos infectados também contribuem para a subnotificação dos casos. Em conjunto, estes fatores colocam em risco a economia e o desenvolvimento saudável dos rebanhos, além de ser prejudicial à saúde humana e ao meio ambiente. ${ }^{24}$

TABELA 1: Número de casos de doenças notificados ao Serviço Veterinário Oficial nos anos de 2017, 2018 e 2019

\begin{tabular}{|c|c|c|c|c|c|}
\hline \multirow{2}{*}{ Doenças } & \multicolumn{3}{|c|}{ Ano } & \multirow{2}{*}{ Média* } & \multirow{2}{*}{ Total } \\
\hline & 2017 & 2018 & 2019 & & \\
\hline Tuberculose Bovina & 4581 & 4907 & 7172 & 5553 & 16660 \\
\hline Anemia Infecciosa Equina & 5189 & 5802 & 4812 & 5268 & 15803 \\
\hline Brucelose (B. abortus) & 3445 & 3807 & 6982 & 4745 & 14234 \\
\hline Raiva & 1170 & 1063 & 1291 & 1175 & 3524 \\
\hline Peste suína clássica & - & 811 & 746 & 779 & 1557 \\
\hline Mormo & 131 & 29 & 74 & 78 & 234 \\
\hline Estomatite vesicular & 17 & 7 & 45 & 23 & 69 \\
\hline Doença de Aujeszky & - & 46 & - & 46 & 46 \\
\hline Língua Azul & 23 & 10 & 5 & 13 & 38 \\
\hline Scrapie & 16 & - & 1 & 9 & 17 \\
\hline Infestação por Aethina tumida & 16 & - & - & 16 & 16 \\
\hline Febre do Oeste do Nilo & - & 10 & 3 & 7 & 13 \\
\hline Encefalopatia espongiforme bovina & - & - & 1 & 1 & 1 \\
\hline
\end{tabular}

Dentre as doenças da Lista da OIE no Brasil, ou seja, doenças de importância mundial, monitoradas pela OIE 25 , um total de 43 estão presentes no território nacional ou apresentam infecção limitada a uma ou mais zonas, incluindo leishmaniose, raiva, brucelose (B. abortus), leucose enzoótica bovina e mormo. Essas doenças podem causar perda reprodutiva, redução da produtividade, morte e/ou limitar a comercialização e exportação de animais e produtos. ${ }^{1,24,20,26}$ Por sua vez, 32 doenças da Lista da OIE nunca foram registradas no Brasil e outras 17 são consideradas ausentes, com última ocorrência em 2019 ou anteriormente. 25

\section{Benefícios da notificação transparente e oportuna de doenças animais}

A agropecuária brasileira é uma das maiores do mundo, destacando-se na criação de gado ( $1^{\circ}$ maior rebanho do mundo), perus ( $2^{\circ}$ maior criação do mundo), suínos ( $3^{\circ}$ maior criação do mundo), cavalos e mulas ( $3^{\circ}$ e $2^{\circ}$ maior criação do mundo, respectivamente), galinha ( $4^{\circ}$ maior criação do mundo), entre outros. ${ }^{21} \mathrm{~A}$ produção é muitas vezes destinada ao mercado interno e externo. Além dos animais domésticos, o Brasil possui uma grande variedade de espécies de animais silvestres que podem ser reservatórios de doenças zoonóticas. A Lei de Proteção à Fauna ( ${ }^{\circ} 5.197$, de 3 de janeiro de 1967 ) proíbe a criação de animais silvestres. No entanto, muitas pessoas descumprem a lei e criam, em suas casas, répteis, aves e mamíferos silvestres, principalmente em grandes centros urbanos, aumentando os riscos de humanos contraírem doenças zoonótica. ${ }^{11}$

Devido a sua biodiversidade, em especial na região amazônica, o Brasil tem sido apontado como um centro propício ao surgimento de novas doenças animais e humana. 27,22 Muitas doenças animais são consideradas barreira sanitária no comércio internacional e, sua presença em determinadas regiões, implica elevados investimentos para seu controle e graves prejuízos em casos de surto. Portanto, surtos de doenças animais podem ocasionar danos 
econômicos e sociais irreparáveis para a agropecuária brasileira. ${ }^{28,8}$

Mais de $20 \%$ das perdas na produção animal mundial são atribuídas as doenças animais. ${ }^{27}$ Como mencionado anteriormente, a maioria destas doenças podem afetar a saúde humana e causar prejuízos sociais e econômicos. As zoonoses emergentes são uma ameaça crescente à saúde global e causam milhões de mortes todos os anos, além de prejuízos econômicos de centenas de bilhões de dólares. ${ }^{14}$ Os dados do SVO são usados para monitorar a prevalência de doenças, a eficácia de programas de controle e, após a erradicação da doença, para documentar a ausência contínua de doença em uma determinada região ou zona. ${ }^{17}$ Estes dados também são base para a certificação zoossanitária nacional junto a organizações internacionais e países ou blocos econômicos com os quais o Brasil mantém relações comerciais.

A melhoria contínua do processo de notificação de doenças da OIE teve efeitos positivos, tanto em termos de prevenção, controle e erradicação de doenças animais em nível global, quanto por facilitar o comércio internacional de animais, seus produtos e subprodutos. ${ }^{6}$ Portanto, realizar a notificação da suspeita ou ocorrência de doença é obrigatória para qualquer cidadão, bem como para todo profissional que atue na área de diagnóstico, ensino ou pesquisa em saúde animal.

\section{Mecanismos da notificação}

Existem vários meios para se realizar a notificação de doenças ao SVO, incluindo o contato direto (presencial), por telefone (números disponibilizados pelo MAPA) ou por meio eletrônico (envio de fax ou e-mail). 7,19 Uma forma direta e simples é a notificação pelo Sistema Brasileiro de Vigilância e Emergências Veterinárias (e-SISBRAVET), que direciona a notificação à unidade veterinária local responsável pelo atendimento no município de localização dos animais suspeitos. A página virtual do SIZ contém as diretrizes para a notificação de doenças e, por meio do link (http://sistemasweb.agricultura.gov.br/pages/ SISBRAVET.html), permite acesso direto a página virtual do e-SISBRAVET. ${ }^{8}$

$\mathrm{Na}$ página do e-SISBRAVET, estão disponíveis e-mail para contato, para esclarecimento de dúvidas, solicitação de demandas e reclamações, manual do usuário, vídeos tutoriais e demais informações para o processo de notificação. Portanto, o e-SISBRAVET é um sistema eletrônico informatizado, específico para gestão dos dados obtidos na vigilância passiva em saúde animal, desenvolvido para o registro e acompanhamento das notificações de suspeitas de doenças e das investigações realizadas pelo SVO. ${ }^{8,19}$

Ao tomar conhecimento de uma nova notificação, o SVO dos Estados (SVEs), representado pelas Unidades Veterinárias Locais (UVLS) e escritórios de atendimento à comunidade, irão realizar a investigação e tomar providências necessárias, conforme previsto na legislação. O processo de investigação é acompanhado pelas instâncias centrais do MAPA (Superintendências Federais de Agricultura nas Unidade Federativas e Departamento de Saúde Animal, em Brasília), até a confirmação ou exclusão de doenças de notificação imediata das categorias 1, 2 e 3, que requerem aplicação de medidas de controle ou erradicação pelo SVO.

\section{Programa de Controle de Zoonoses}

Prever o surgimento ou a volta de epidemias é difícil. ${ }^{11}$ Instituições de saúde pública com estrutura setorizada e baixa integração entre as equipes de campo, práticas antiquadas, como sistema de armazenamento baseado em papel e agentes 
de saúde mal qualificados, têm capacidade limitada para conduzir vigilância precisa e planejar intervenções eficazes e oportunas das doenças. ${ }^{22}$ As medidas de segurança e controle de doenças devem ser embasadas em conhecimento amplo sobre patógenos, hospedeiros, vetores e fatores que favorecem o ciclo da doença.

As zoonoses podem ser diretas, quando o patógeno é transmitido diretamente de animais para humanos, ou indiretas, quando um vetor é necessário para transportar o patógeno. 150 estabelecimento e a manutenção do ciclo de transmissão das zoonoses podem ser favorecidos por diversos fatores ambientais e socioeconômicos, tais como domesticação e interação com animais silvestres, migração, urbanização, desmatamento e perda de biodiversidade, que facilitam a presença de vetores, reservatórios e parasitas, facilitando ainda mais a ocorrência da doença. ${ }^{11,27,29}$

Uma abordagem mais eficaz para prevenção e controle de doenças zoonóticas exigirá uma visão ampla da medicina, que enfatize a tomada de decisão baseada em evidências e integre princípios ecológicos e evolutivos de fatores animais, humanos e ambientais. ${ }^{14,22}$ Portanto, os mecanismos ecológicos, evolutivos, sociais, econômicos e epidemiológicos que afetam a persistência e emergência das zoonoses precisam ser melhor compreendidos, para informar políticas baseadas em evidências, práticas e vigilância direcionadas de doenças zoonóticas, e esforços de prevenção e controle. ${ }^{14}$

A One Health (do inglês, "saúde única") é uma estratégia sinérgica para expandir as colaborações interdisciplinares de cuidados da saúde para humanos, animais e meio ambiente. ${ }^{22}$ Grande parte da literatura abordando o conceito One Health está relacionada à saúde animal com enfoque nas doenças transfronteiriças, que são zoonóticas por natureza e, portanto, transmissíveis e infecciosas para humanos. 5 De fato, há uma conexão entre a saúde humana, animal e ambiental. Portanto, as doenças zoonóticas emergentes e reemergentes podem ser mais bem compreendidas e controladas com a perspectiva One Health (Figura 2). ${ }^{15,22}$

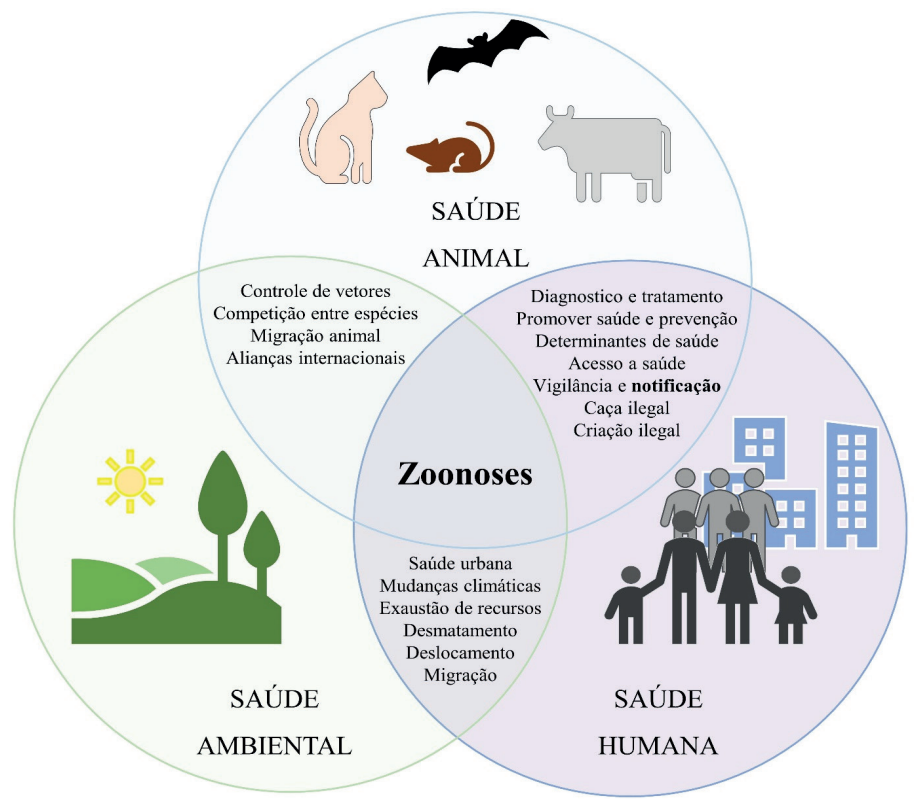

FIGURA 2: Abordagem “One Health” para lidar com doenças zoonóticas. Fonte: adaptado de Marbán-Castro, Mattar \& González 15 
O Centro de Controle de Zoonoses de Foz do Iguaçu, Paraná, adotou a abordagem One Health e passou de uma estrutura arcaica e setorizada para um programa de vigilância moderno e oportuno, abrangendo doenças zoonóticas, lesões de animais peçonhentos e epidemiologia das doenças transmitidas por vetores. 30,22

A implementação completa da abordagem foi baseada em 5 eixos: (1) fusão de equipes de campo setorizadas; (2) adoção de soluções digitais; (3) empoderamento e capacitação permanente dos agentes de saúde; (4) mobilização social; e (5) pesquisas ativas. Com a adoção dessa abordagem, as notificações relacionadas a zoonoses e animais peçonhentos aumentaram 10 e 21 vezes, respectivamente, no município de Foz do Iguaçu. O banco de dados, obtido com as notificações, é atualizado diariamente e cria mapas em tempo real para apoiar decisões oportunas. A adoção da abordagem One Health aumentou a preparação para doenças endêmicas e ameaças reemergentes e emergentes, como a Síndrome Respiratória Aguda Grave por Coronavírus (SARS-CoV-2). ${ }^{22}$

\section{CONSIDERAÇÕES FINAIS}

A Instrução Normativa $n^{\circ} 50$, de 24 de setembro de 2013, dispõe sobre uma lista de doenças de notificação obrigatória (DNO) no Brasil. A lista contempla, atualmente, um total de 141 doenças, incluindo as DNO para a World Organisation for Animal Health. Das doenças da lista da OIE no Brasil, 43 estão presentes ou com infecção limitada a uma ou mais zonas. $\mathrm{Na}$ base de dados zoossanitários do Brasil, apenas 13 doenças tiveram casos notificados nos últimos 3 anos disponíveis (2017, 2018 e 2019). Dentre essas, destacam-se a tuberculose bovina, anemia infecciosa equina, brucelose (B. abortus), raiva e peste suína clássica como
Essa visão ampla, proporcionada na abordagem One Health, é essencial para o desenvolvimento bem-sucedido de políticas e práticas que reduzam a probabilidade de emergência futura de zoonoses, vigilância direcionada, prevenção estratégica e envolvimento de parceiros fora da comunidade médica para ajudar a melhorar os resultados de saúde e reduzir as ameaças de doenças. ${ }^{14}$

Portanto, o futuro da prevenção e controle das doenças animais, sobretudo as zoonoses, depende de uma abordagem integrada, envolvendo veterinários, donos de animais, produtores rurais e autoridades sanitárias, a fim de reduzir o risco de infecção animal e, consequentemente, a transmissão para humanos. ${ }^{31} \mathrm{~A}$ notificação é uma ferramenta chave para controlar, evitar, ou erradicar uma doença, mas deve estar associada a uma rede de cooperação com ações estratégicas em vigilância, pesquisa, comunicação e capacitação 27 , intervindo sobre os fatores de risco associados a prevalência das doenças endêmicas e evitando as doenças transfronteiriças. doenças de maior notificação média no país.

Pesquisas indicam elevado índice de subnotificação e falta de conhecimento sobre o processo notificação. $O$ processo de notificação é simples e acessível a todos. Instruções para notificação foram aqui apresentadas. A conexão entre a saúde humana, animal e ambiental, e a necessidade de estudar as doenças em seu contexto biológico, ecológico, médico e econômico, são apontados como mecanismo para prevenção e controle de doenças, de forma a promover e garantir a saúde nacional e globalmente. 


\section{REFERÊNCIAS BIBLIOGRÁFICAS}

1. Bronner $A$, Hénaux $V$, Fortané $N$, Hendrikx $P$, Calavas $D$. Why do farmers and veterinarians not report all bovine abortions, as requested by the clinical brucellosis surveillance system in France?. BMC Vet. Res. 2014 Dec; 10(1): 1-2.

2. Chowdhury S, Aleem MA, Khan MS, Hossain ME, Ghosh S, Rahman MZ. Major zoonotic diseases of public health importance in Bangladesh. Vet. Med. Sci. 2021 Jan 1.

3. Coka Z, Chulayo AY. The economic impact of animal disease outbreaks: management \& technology. Stock. 2019 Jan; 9(1): 52-3.

4. Osofsky SA. The global burden of (how we manage) animal disease: Learning lessons from southern Africa. J. Wildl. Dis. 2019 Oct; 55(4): 75557.

5. Torres-Velez F, Havas KA, Spiegel K, Brown C. Transboundary animal diseases as re-emerging threats-Impact on one health. In Semin. Diagn. Pathol. 2019 May; 36(3): 193-96. WB Saunders.

6. Caceres P, Tizzani P, Ntsama F, Mora R. The World Organisation for Animal Health: notification of animal diseases. Rev. Sci. Tech. (International Office of Epizootics). 2020 Apr; 39(1): $289-97$.

7. World Organisation for Animal Health. Acesso em 28 dez 2020. Disponível em: https://www. oie.int/

8. MAPA. Sistema de Informação em Saúde Animal. Acesso em 08 jan 2021. Disponível em: https://www.gov.br/agricultura/pt-br/assuntos/ sanidade-animal-e-vegetal/saude-animal/ epidemiologia/portugues/sistema-informacaosaude-animal.

9. Brasil. Instrução Normativa no 50, de 24 de setembro de 2013. Diário Oficial da República Federativa do Brasil. 2013.
10. Zepeda C, Salman M, Thiermann A, Kellar $\mathrm{J}$, Rojas $\mathrm{H}$, Willeberg $\mathrm{P}$. The role of veterinary epidemiology and veterinary services in complying with the World Trade Organization SPS agreement. Prev. Vet. Med. 2005 Feb; 67(23): $125-40$.

11. Silva JC. Zoonoses e doenças emergentes transmitidas por animais silvestres. ABRAVAS. 2004: 1-4.

12. Han BA, Kramer AM, Drake JM. Global patterns of zoonotic disease in mammals. Trends Parasitol. 2016 Jul; 32(7): 565-77.

13. Wang LF, Crameri G. Emerging zoonotic viral diseases. Rev. Sci. Tech. 2014 Aug; 33(2): 569-81.

14. Karesh WB, Dobson A, Lloyd-Smith JO, Lubroth J, Dixon MA, Bennett M, Aldrich S, Harrington T, Formenty P, Loh EH, Machalaba CC. Ecology of zoonoses: natural and unnatural histories. The Lancet. 2012 Dec; 380(9857):193645.

15. Marbán-Castro E, Mattar S, González TM. Reemerging zoonoses with "One Health" approach. Rev. MVZ Cordoba. 2019; 24(3): 728084.

16. Peace N. Impact of climate change on insects, pest, diseases and animal biodiversity. IJESNR. 2020; 23(4): 165-67.

17. Perry BD, Grace D, Sones K. Current drivers and future directions of global livestock disease dynamics. Proc. Natl. Acad. Sci. 2013 Dec; 110(52): 20871-77.

18. de Araújo GM, de Lima PR, Simplício KM, da Silva Júnior JL, dos Santos KA, Silva AG. Sistema de Informação em Saúde Animal: percepção de estudantes, profissionais de instituições de ensino da Medicina Veterinária e de veterinários autônomos do Estado de Sergipe quanto à 
notificação obrigatória de doenças ao Serviço Veterinário Oficial. Braz. J. Dev. 2020 Oct; 6(10): 81826-39.

19. MAPA. Coordenação de Informação e Epidemiologia - Saúde Animal. Acesso em 05 jan 2021. Disponível em: http://indicadores. agricultura.gov.br/saudeanimal/index.htm .

20. Batista HR, Passos CT, Nunes Neto OG, Sarturi C, Coelho AP, Moreira TR, et al. Factors associated with the prevalence of antibodies against Brucella abortus in water buffaloes from Santarém, Lower Amazon region, Brazil. Transbound Emerg. Dis. 2020 Jul; 67: 44-8.

21. FAOSTAT. Food and Agriculture Organization. Acesso em 17 de fev de 2021. Disponível em: http://www.fao.org/faostat/en/\#data.

22. Leandro AD, Lopes RD, Martins CA, Rivas AV, da Silva I, Galvão SR, et al. The adoption of the One Health approach to improve surveillance of venomous animal injury, vector-borne and zoonotic diseases in Foz do Iguaçu, Brazil. PLOS Negl. Trop. Dis. 2021 Feb;15(2): eooog109.

23. Minervino AH, Soares HS, Barrêto-Júnior RA, Neves KA, Morini AC, Ortolani EL, etal. Antibodies against brucella abortus and Leptospira spp. in captive mammals in the states of pará and rio grande do norte, brazil. J. Zoo Wildl. Med. 2018 Jun; 49(2): 355-60.

24. El-Diasty M, Wareth G, Melzer F, Mustafa $S$, Sprague LD, Neubauer $H$. Isolation of Brucella abortus and Brucella melitensis from seronegative cows is a serious impediment in brucellosis control. Vet. Sci. 2018 Mar; 5(1): 28.
25. Situação sanitária das doenças de animais terrestres - BRASIL, 2020. Acesso em 12 de mar de 2021. Disponível em: https://www.gov.br/ agricultura/pt-br/assuntos/sanidade-animal-evegetal/saude-animal/arquivos-sisa/Situacao_ zoossanitaria_WAHID_Brasil_2020_1.pdf.

26. Pineda $P$, Deluque A, Peña $M$, Diaz OL, Allepuz A, Casal J. Descriptive epidemiology of classical swine fever outbreaks in the period 2013-2018 in Colombia. PloS one. 2020 Jun 17; 15(6): e0234490.

27. Zanella JR. Zoonoses emergentes e reemergentes e sua importância para saúde e produção animal. PAB. 2016 May; 51(5): 510-09. 28. Garcia DC, Sá CV, McManus CM, Melo CB. Impactos do surto de febre aftosa de 2005 sobre as exportações de carne bovina Brasileira. Ci. Anim. Bras. 2015 Dec;16(4): 525-37.

29. Buzanovsky LP, Sanchez-Vazquez MJ, MaiaElkhoury AN, Werneck GL. Major environmental and socioeconomic determinants of cutaneous leishmaniasis in Brazil-a systematic literature review. Rev. Soc. Bras. Med. Trop. 2020; 53.

30. Dias RC, Thomaz-Soccol V, Pasquali AK, Alban SM, Fendrich RC, Pozzolo EM, et al. Variables associated with the prevalence of anti-Leishmania spp. antibodies in dogs on the tri-border of Foz do Iguaçu, Paraná, Brazil. Rev. Bras. Parasitol. Vet. 2018 Sep; 27(3): 338-47.

31. Cortes S, Vaz Y, Neves R, Maia C, Cardoso L, Campino L. Risk factors for canine leishmaniasis in an endemic Mediterranean region. Vet. Parasitol. 2012 Oct 26;189(2-4): 189-96. 